\title{
Hígado y terapia antituberculosa
}

\author{
MIGUEL AGUAYO C.* y JUAN CARLOS RODRÍGUEZ D.*
}

\section{Hepatotoxicity of antituberculosis therapy}

Liver toxicity in patients being treated with antituberculosis drugs is relatively uncommon, although transient elevation of liver enzymes is very common. Probably because of this there is not a good definition for liver toxicity. There are conditions in which hepatotoxicity is more frequent, such as elderly patients, alcohol consumption, malnutrition, use of certain drugs, and hypoalbuminemia. Pirazinamide, isoniazid and rifampicin are the antituberculosis drugs more commonly involved in liver toxicity. In this article we analyze the clinical problem of hepatotoxicity of antituberculosis therapy and propose the management of different situations. We discuss when a drug administration should be discontinued, how liver damage should be assesed and which alternative drugs should be used during the antituberculosis treatment.

Key words: Antituberculosis therapy; hepatotoxicity; Pirazinamide, Isoniazid; Rifampicin.

\section{Resumen}

La toxicidad hepática en pacientes tratados con drogas antituberculosas es relativamente infrecuente, Probablemente debido a este hecho no contamos con una buena definición de toxicidad hepática. Existen algunas condiciones de los enfermos en que la hepatotoxicidad es más frecuente, tales como pacientes envejecidos, bebedores de alcohol, desnutrición, uso de ciertas drogas e hipoalbuminemia. Las drogas antituberculosas más frecuentemente involucradas en hepatotoxicidad son la pirazinamida, la isoniacida y la rifampicina. En este artículo analizamos el problema clínico de la hepatotoxicidad de la terapia antituberculosa y proponemos el manejo de diferentes situaciones. Discutimos cuando se debe suspender la administración de una droga, cómo se debe evaluar el daño hepático y qué drogas alternativas pueden usarse durante el tratamiento de la tuberculosis.

Palabras clave: Terapia antituberculosa; hepatotoxicidad; Pirazinamida; Isoniacida; Rifampicina.

\section{Introducción}

Entre los principales efectos adversos del tratamiento antituberculoso actual están las reacciones cutáneas, las molestias gastrointestinales y la hepatotoxicidad (HPD).

La frecuencia de las reacciones adversas es variable. De acuerdo a las cifras internacionales varía entre 0,07 a $11,9 \%$; la mayoría de las veces no requiere la suspensión de los medicamentos.

En un meta-análisis reciente de quimioprofilaxis con isoniacida para tuberculosis latente, en 1.861 participantes se encontró una baja incidencia de HPD, con una tasa media de $1,8 \%$. La incidencia en mayores de 35 años fue de $1,7 \%$ y en menores de esta edad de $0,2 \%$.
No existe consenso en la definición de la hepatotoxicidad por drogas antituberculosas, ya que es sabido que algunas de ellas producen alteraciones transitorias de las enzimas hepáticas. El límite para definir hepatotoxicidad no está claro y tampoco cuáles son las enzimas para medirlo.

Los consensos de la Sociedad Británica del Tórax (BTS), de la Sociedad Americana del Tórax (ATS), la guía de la Sociedad Respiratoria de Europa (ERS) y las normativas de la OMS, coinciden en que lo más importante como indicador de toxicidad son los síntomas de hepatitis. Sin embargo, la monitorización de las enzimas hepáticas pareciera ser útil en la detección de casos asintomáticos que podrían desarrollar insuficiencia hepática de continuar con la terapia.

* Servicio de Medicina, Instituto Nacional del Tórax. Santiago de Chile. 
Hyman Zimmerman describió lo que hoy se denomina ley de Hy, que señala que un aumento de 3 veces de la ALT (alanina- aminotransferasa) y de 2 veces de la bilirrubina total, se asocia a $10 \%$ de desarrollo de insuficiencia hepática con caída de la protrombina en 24 a 36 horas, de continuar con la medicación.

Para unificar criterios, revisamos las guías internacionales y la literatura anglosajona, con la idea de hacer una proposición consensuada para el manejo de los enfermos con hepatotoxicidad secundaria al empleo de drogas antituberculosas.

\section{Previo al inicio de la terapia antituberculosa}

$\mathrm{Al}$ inicio de la terapia antituberculosa es muy importante considerar los eventuales factores de riesgo para el desarrollo de HPD.

\subsection{Antecedentes clínicos}

Edad. A mayor edad, mayor es el riesgo de HPD. El mayor riesgo se hace evidente desde los 35 años y aumenta sobre los 60 años.

Embarazo. El tercer trimestre y los tres primeros meses del puerperio son los períodos en que hay mayor riesgo desarrollar HPD.

Alcohol. El consumo de alcohol aumenta al doble la incidencia de HPD y por ello está proscrito durante todo el tiempo que dure la terapia antituberculosis.

Drogas. Existen algunas drogas que aumentan significativamente el riesgo de hepatotoxicidad: las antifúngicas, la carbamazepina, el metrotrexate, el acetaminofeno, y la sulfasalazina.

Co-infección VIH. Algunos estudios la han relacionado con un mayor riesgo HPD, pero otros no han demostrado similar asociación. En relación a la terapia antirretroviral y hepatitis por drogas, dependiendo de los medicamentos utilizados puede verse un aumento de la incidencia que va desde 2 a $18 \%$, dependiendo de la serie. El esquema de quimioprofilaxis con rifampicinapirazinamida sólo está aprobado para pacientes VIH positivos, ya que en los VIH negativos el porcentaje de hepatotoxicidad es demasiado alto. Se desconoce la razón de esta menor toxicidad hepática en los VIH $(+)$.

Hepatitis. La presencia de una hepatitis activa puede aumentar la hepatotoxicidad por isoniacida. Incluso la infección con virus C, por sí sola aumenta este riesgo. Si a esto se asocia el consumo de alcohol las posibilidades de hepatotoxicidad se elevan en forma importante.

Desnutrición. En pacientes desnutridos, con índice de masa corporal bajo, especialmente en los portadores de hipoalbuminemia o en aquellos que tienen deficiencias vitamínicas, la toxicidad hepática también aumenta.

\subsection{Laboratorio}

Se recomienda obtener niveles basales de enzimas hepáticas ALT, aspartato transaminasa (AST) y bilirrubina total en los grupos de riesgo mencionados antes de iniciar el tratamiento. Respecto a otras enzimas como $\gamma$-glutamil transpeptidasa (GGT) y fosfatasa alcalina no hay reportes sobre la utilidad de su medición y su significado clínico es más bien controvertido.

En caso que las enzimas mencionadas estén elevadas, antes del inicio de la terapia, se sugiere:

- Realizar estudio funcional hepático completo, que incluya una ecografia abdominal y marcadores para los virus de hepatitis A, B, C y también VIH.

- Controlar semanalmente las enzimas y no iniciar tratamiento habitual antes que éstas se normalicen o bajen por lo menos hasta un valor no superior a tres veces el normal. En caso que la tuberculosis del paciente sea muy avanzada o muy grave es recomendable iniciar un esquema que tenga mínimo riesgo de toxicidad hepática, mientras se normalizan las alteraciones. Se sugiere en estas condiciones indicar la asociación estreptomicina-etambutol.

- Cuando las enzimas se hayan normalizado o hayan bajado a un nivel inferior a 3 veces el valor normal, se sugiere re-iniciar la terapia. El control de enzimas en esta situación debe ser inicialmente muy frecuente. En caso que vuelvan a subir en forma significativa o aparezcan síntomas, deberá cambiarse al esquema de daño hepático.

Cuando se inicia terapia con enzimas hepáticas normales, se sugiere:

- Educar al enfermo sobre los posibles efectos colaterales y advertirle de los eventuales riesgos, especialmente asociados al consumo de otros medicamentos hepatotóxicos.

- Educar al enfermo para el reconocimiento de los síntomas de hepatitis (ver monitoreo de la terapia más adelante). Es importante que el paciente sepa que si tiene cualquiera de los síntomas enumerados a continuación debe suspender el tratamiento y consultar médico:

- Dolor abdominal.

- Náuseas.

- Vómitos.

- Malestar general.

- Ictericia. 
- Evitar consumo de alcohol durante el tratamiento antituberculoso. Esta indicación nos parece altamente recomendable y más perentoria en los casos en que haya otros factores de riesgo asociados.

\section{Seguimiento durante la terapia}

Cuando el paciente presente síntomas digestivos es importante controlar las enzimas, lo que no se justifica cuando no hay síntomas.

\subsection{Se considera hepatitis por drogas antituberculosas cuando:}

- ALT/AST es mayor de 3 veces el límite superior normal (LSN), más síntomas.

- ALT/AST es mayor a 5 veces LSN en ausencia de síntomas.

- ALT/AST es mayor a 3 veces LSN y la bilirrubina total 2 veces LSN.

- Aumentos menores de 3 veces LSN deben ser controlados a la semana para observar la tendencia. Luego cada 2 semanas hasta que vuelvan a lo normal o cumplan criterios de hepatitis.

- Cada vez que se haga el diagnóstico de HPD deben descartarse otras causas de hepatitis. Averiguar la existencia de otras drogas hépatotóxicas, solicitar los marcadores virales y eventualmente una ecografía abdominal.

- La medición mensual de enzimas hepáticas, aunque no ha demostrado que disminuya la mortalidad de la hepatitis por drogas antituberculosas, puede ser recomendable en casos especiales para su detección precoz.

\subsection{Reinicio del tratamiento: Dos situaciones clínicas aparecen en el paciente que presenta hepatitis por drogas}

a) Si el enfermo está en riesgo vital por la tuberculosis (meníngea, miliar, pericárdica, pulmonar muy avanzada) y la suspensión de la terapia puede desencadenar la muerte, se sugiere en conjunto con el especialista en tuberculosis iniciar esquema sin hépato-tóxicos mientras se recupera la función hepática, idealmente al menos con 3 drogas. Nuestra sugerencia es asociar etambutol, estreptomicina y una fluoroquinolona, hasta recuperar su función hepática.

b) Si el paciente no está con riesgo vital y da tiempo para esperar la recuperación completa de las alteraciones hepáticas, nuestra sugerencia es proceder a la re-introducción de la medicación según el siguiente esquema:

Día 1: Isoniacida $50 \mathrm{mg}$, aumentar hasta 300 mg en 3 días.

Día 3: Rifampicina $75 \mathrm{mg}$ aumentar hasta 600 mg en 4 días.

Una vez alcanzada las dosis plenas de isoniacida y rifampicina, si no hay evidencias de daño hepático, se pueden agregar las otras drogas. Sin embargo, el Programa Nacional de Control de la Tuberculosis (PNT), no recomienda la reintroducción de pirazinamida, por ser la droga que más frecuentemente induce toxicidad hepática.

Hay que medir la función hepática diariamente hasta completar las dosis completas de los medicamentos; luego una vez a la semana durante un mes y luego mensualmente.

Cuando no se emplea pirazinamida debe prolongarse a 9 meses el esquema primario.

\section{Situaciones especiales}

\subsection{Paciente con enfermedad hepática previa}

En los enfermos con hepatopatía crónica estable se recomienda la medición basal de enzimas hepáticas y el inicio del esquema con 3-4 drogas, evitando el uso de pirazinamida.

Se sugiere monitoreo de las enzimas dos veces por semana los primeros quince días, luego cada 2 semanas por 2 meses, y luego mensualmente hasta terminar el tratamiento, con el objetivo de detectar precozmente una toxicidad hepática por drogas.

En pacientes con insuficiencia hepática descompensada se recomienda la asociación de estreptomicina, isoniacida y etambutol (Normas PNT 2005). Si el riesgo es muy alto se puede reemplazar la isoniacida por una fluoroquinolona, luego de una evaluación por especialista o por el encargado del PNT.

\subsection{Hepatitis en el curso de quimioprofilaxis}

También puede aparecer una hepatitis en el curso de una quimioprofilaxis, con una frecuencia que varía con las diferentes drogas: $0,5 \%$ a $1 \%$ con isoniacida, $1-2 \%$ con rifampicina, $2 \%$ con rifampicina-isoniacida y $8-13 \%$ con la combinación de pirazinamida-rifampicina (esquema que no es recomendable en pacientes no VIH).

$\mathrm{Al}$ inicio de una quimioprofilaxis es igualmente importante la educación del enfermo sobre los eventuales efectos adversos que pueden presentarse, tal como se recomienda antes de iniciar un tratamiento antituberculoso. También debe insistirse en la abstinencia de alcohol, siendo discutible la indicación de monitorizar rutinariamente las enzimas hepáticas en estos casos. 
Cuando aparezcan criterios de hepatitis se debe proceder de manera similar a lo señalado para los pacientes con terapia antituberculosa habitual. Además de la suspensión inmediata del medicamento, es necesario descartar otras causas de hepatitis. La reintroducción de la isoniacida se deberá plantear sólo si el riesgo de desarrollo de tuberculosis, justifica la mantención de una quimioprofilaxis en esas condiciones.

\subsection{Drogas antituberculosis y trasplante hepático}

La realidad nacional e internacional de avances en hepatología ha hecho que algunos pacientes con daño hepático crónico accedan a la indicación de trasplante como medida terapéutica. Es un grupo de especial riesgo respecto al potencial desarrollo de falla hepática secundario a drogas o descompensación de patologías previas.

No hay estudios randomizados sobre quimioprofilaxis ni terapia antituberculosa en estos enfermos; por lo tanto, las conductas a seguir se toman sólo en base a la opinión de expertos o guías internacionales.

En reportes internacionales el Grupo RESITRA (Red española de investigación de la infección en el trasplante) reporta una prevalencia de $0,47 \%$ de tuberculosis activa en este grupo de pacientes, con una incidencia de 308 por 100.000 , muy en exceso respecto a la población general.

El riesgo de desarrollo de tuberculosis está asociado a:

- Tratamiento inmunosupresor empleado.

- Grado de inmunosupresión.

- Intensificación de inmunosupresión en rechazo agudo.

- Historia de exposición a M. tuberculosis.

- PPD positivo.

- TBC antigua no tratada.

- Condiciones clínicas.

- Diabetes Mellitus.

- Insuficiencia renal crónica en hemodiálisis

- Virus hepatitis C.

- Infecciones coexistentes.

Durante el estudio pre-trasplante se recomienda medir PPD, que se considera positivo si es mayor a $5 \mathrm{~mm}$.

En enfermos con anergia, dependiendo de la estimación de riesgo de tuberculosis de esa población, se recomienda la utilización ya sea de Elispot o Quantiferón TB Gold para el diagnóstico de infección.

Además, debe obtenerse una historia adecuada de posibles terapias antituberculosas previas completas o incompletas y una radiografía de tórax, con el objeto de descartar una enfermedad activa.

En el caso específico de trasplante hepático se recomienda retardar la administración de quimioprofilaxis hasta después del trasplante, ojalá con función hepática normal y estable. En un estudio que revisó 238 pacientes que recibieron isoniacida durante el período pre-trasplante se reportó $0 \%$ de tuberculosis activa después de trasplante versus un $8,2 \%$ en los que no lo recibieron. Se recomienda analizar la decisión caso a caso, idealmente en conjunto el neumólogo y el hepatólogo.

En pacientes trasplantados existe consenso en que se debe indicar profilaxis en las siguientes situaciones:

1. Conversión de PPD reciente.

2. Antecedentes de tuberculosis mal tratada.

3. Contacto con enfermo de tuberculosis activa.

4. Lesiones residuales en la radiografía de tórax en un paciente no tratado.

5. Intensificación de la inmunosupresión en el manejo de rechazo agudo en casos PPD positivos sin terapia previa.

En estos enfermos se ha reportado la aparición de hasta $6 \%$ de hepatitis por isoniacida, por lo que deben ser estrechamente monitorizados durante su administración.

En los casos de trasplantados que desarrollan una tuberculosis activa se ha encontrado una mortalidad a 26 meses de un 31\%. En el 67\% de los casos la tuberculosis es extrapulmonar, con formas miliares en $27 \%$.

Una vez confirmada una tuberculosis en un paciente con trasplante hepático debe ser tratado en forma activa con tres drogas. Se sugiere la asociación de etambutol, estreptomicina y una fluoroquinolona. Según la función hepática puede agregarse isoniacida; en cambio, el empleo de rifampicina en general se debe evitar o administrarse con mucho cuidado por su interferencia con el metabolismo de las drogas inmunosupresoras. No se recomienda el uso de pirazinamida.

Se requiere de mayor experiencia y más estudios para definir la terapia óptima en estos casos. Por el momento, la indicación de quimioprofilaxis (tratamiento de la tuberculosis latente) es controvertida y debe ser evaluada caso a caso.

\section{Conclusiones}

El compromiso hepático por drogas debe ser reconocido por los médicos, enfermeras y personal de salud que está en relación con enfermos tuberculosos. 
Un alto nivel de sospecha y la educación de los enfermos son imprescindibles para evitar casos potencialmente fatales.

El manejo de los pacientes que desarrollan hepatotoxicidad por drogas requiere de un manejo especializado idealmente por un neumólogo o por el médico a cargo del PNT entrenado.

\section{Bibliografía}

1.- WHO. Treatment of tuberculosis: guidelines -4 th ed. WHO/HTM/TB/2009.420. 1. Antitubercular agents administration and dosage. 2. Tuberculosis, Pulmonary -drug therapy. 3. National health programs. 4. Patient compliance. 5. Guidelines. Intl .World Health Organization. Stop TB Dept.

2.- Joint Tuberculosis Committee of the British Thoracic Society * Chemotherapy and management of tuberculosis in the United Kingdom: recommendations 1998. Thorax 1998; 53: 536-48.

3.- SAUKKONEN J J, COHN D L, JASMER R M, SCHENKER S, JEREB J A, NOLAN C M, et al. An Official ATS Statement: Hepatotoxicity of Antituberculosis Therapy. Am J Respir Crit Care Med 2006; 174: 935-52.

4.- TOSTMANN A, BOEREE M J, AARNOUTSE R B, DE LANG W C M, VAN DER VEN A J A M, DE-
KHUIJZEN R. Antituberculosis drug-induced hepatotoxicity: Concise up-to-date review. J Gastroenterol Hepatol 2008; 23: 192-202.

5.- SHARMA S K, SINGLA R, SARDA P, MOHAN A, MAKHARIA G, JAYASWAL A, et al. Safety of 3 Different Reintroduction Regimens of Antituberculosis Drugs after Development of Antituberculosis Treatment-Induced Hepatotoxicity Clin Infect Dis 2010; 50: 833-9.

6.- BTS recommendations for assessing risk and for managing Mycobacterium tuberculosis infection and disease in patients due to start anti-TNF- $\alpha$ treatment British Thoracic Society Standards of Care Committee. Thorax 2005; 60: 800-5.

7.- AGUADO J M, TORRE-CISNEROS J, FORTÍN J, BENITO N, MEIJE Y. DOBLAS A, et al. Documento de consenso para el tratamiento de la tuberculosis en pacientes con trasplante de órgano sólido. España Enf Infecc Microbiol Clin 2009; 27: 465-73.

8.- HOLTY J E C, GOULD M K, MEINKE L, KEEFFE E B, RUOSS S J. Tuberculosis in Liver Transplant Recipients: A Systematic Review and Meta-Analysis of Individual Patient Data. Liver Transplantation 2009; 15: 894-906.

9.- KUNST H, KHAN K S. Age-related risk of hepatotoxicity in the treatment of latent tuberculosis infection: a systematic review. Intl J Tuberculosis Lung Dis 2010; 14: $1374-81$.

Correspondencia a:

Dr. Juan Carlos Rodríguez Duque

Servicio de Medicina, Instituto Nacional del Tórax.

José Miguel Infante 717.

Providencia, Santiago de Chile.

Fono-fax: 056 - 02 - 5754997

E-mail: jotarodriguez@123.cl 